\title{
Calibration of the distance scale from galactic Cepheids
}

\section{Use of the HIPPARCOS calibration ${ }^{\star}$}

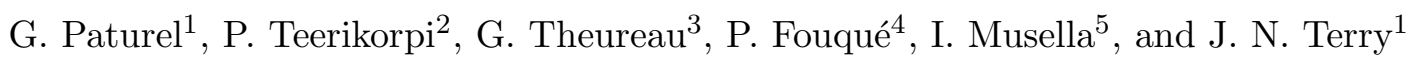 \\ 1 CRAL-Observatoire de Lyon, avenue Charles-André, 69561 Saint-Genis Laval Cedex, France \\ 2 Turku University Observatory, Tuorla, SF 21500 Piikkio, Finland \\ 3 Laboratoire de Physique et de Chimie de l'Environnement, 3A avenue de la Recherche scientifique, \\ 45071 Orleans Cedex 02, France \\ 4 European Southern Observatory, Casilla 19001, 19 Santiago, Chile \\ 5 Osservatorio Astronomico di Capodimonte, via Moiariello 16, 80131 Napoli, Italy
}

Received 8 August 2001 / Accepted 1 March 2002

\begin{abstract}
New estimates of the distances of 36 nearby galaxies is presented. These are based on the calibration of the $V$ - and $I$-band Period-Luminosity relations for galactic Cepheids measured by the HIPPARCOS mission. The distance moduli are obtained in a classical way. The statistical bias due to the incompleteness of the sample is corrected according to the precepts introduced by Teerikorpi (1987).

We adopt a constant slope (the one obtained with LMC Cepheids). The correction for incompleteness bias introduces an uncertainty that depends on each galaxy. On average, this uncertainty is small (0.04 mag) but it may reach $0.3 \mathrm{mag}$. We show that the uncertainty due to the correction of the extinction is small (propably less than $0.05 \mathrm{mag}$ ). The correlation between the metallicity and the morphological type of the host galaxy suggests that we should reduce the application to spiral galaxies in order to bypass the problem of metallicity. We suspect that the adopted PL slopes are not valid for all morphological types of galaxies. This may induce a mean systematic shift of $0.1 \mathrm{mag}$ on distance moduli.

A comparison with the distance moduli recently published by Freedman et al. (2001) shows there is a reasonably good agreement with our distance moduli.
\end{abstract}

Key words. galaxies: distances and redshift - galaxies: stellar content - cosmology: distance scale

\section{Introduction}

We have started a new study of the kinematics of the local universe $(\mathrm{KLUN}+)$ which aims at determining peculiar velocities for nearby galaxies $\left(\approx 100 h^{-1} \mathrm{Mpc}^{-1}\right)$. The radial component of such a peculiar velocity is obtained by subtracting the Hubble flow from the observed radial velocity. This means that the underlying Hubble flow must be determined free of any sort of bias (systematic, distance or direction dependent). The distances are obtained through the Tully-Fisher relation (1977) by combining 21-cm line width measurements (Nançay key-project) with infrared magnitudes (DENIS and 2MASS surveys). The Tully-Fisher relation will be calibrated with some very

Send offprint requests to: G. Paturel, e-mail: patu@obs.univ-lyon1.fr

* The compilation of raw data is only available in electronic form at CDS via anonymous ftp to

cdsarc.u-strasbg.fr $(130.79 .128 .5)$ or via

http://cdsweb.u-strasbg.fr/cgi-bin/qcat?J/A+A/389/19

and on our anonymous ftp-server www-obs.univ-lyon1.fr (pub/base/CEPHEIDES.tar.gz). near galaxies $\left(<25 h^{-1} \mathrm{Mpc}^{-1}\right)$. This calibrating step is very important because it will influence all forthcoming results. For this reason, the distances of these calibrating galaxies must be determined carefully on the basis of the Cepheid Period-Lumionosity relation (hereafter, PL relation) which remains the most accurate method of stellar distance determination. Furthermore, the PL relation itself must be calibrated from geometrical means, i.e. from galactic Cepheids. In a previous paper (Paturel et al. 2002, Paper I) we obtained distances for 36 nearby galaxies by comparing, in a straightforward way (the method of "sosie"), extragalactic Cepheids with galactic Cepheids whose accurate distance moduli are available through the Barnes-Evans method (Gieren et al. 1998; hereafter GFG).

Our present purpose is to calculate the distances through the classical PL relation for the same galaxy sample using our calibration (Lanoix et al. 1999) from the HIPPARCOS satellite (Perryman et al. 1997) which measured geometrical parallaxes for a sample of nearby galactic Cepheids. It has been shown (Pont et al. 1997; Lanoix et al. 1999) that the treatment proposed by Feast \& Catchpole (1997) to correct for the 
Lutz \& Kelker's bias (1973) gives an unbiased calibration of the PL relation. The zero-point calibration is independent of the one used in Paper I.

In Sect. 2 the method of calculation is recalled and applied to the $V$ - and $I$-band measurements described in Paper I. So, distances are obtained for 1840 Cepheids belonging to 36 nearby galaxies. In Sect. 3 we discuss these results.

\section{Application to extragalactic Cepheids}

\subsection{The method of calculation}

The $V$-band magnitude $V$ can be corrected for extinction. The corrected $V_{\mathrm{o}}$ magnitude is given through the classical relation:

$V_{\mathrm{o}}=V-R_{V} E_{(B-V)}$

where $R_{V}$ is the coefficient of the total to the differential extinction (as tabulated e.g. by Cardelli et al. 1989; Caldwell \& Coulson 1987; Laney \& Stobie 1994) and $E_{B-V}$ is the $B-V$ color excess (difference between the observed and the intrinsic $B-V$ ).

Similarly, the $I$-band magnitude can be corrected through the relation:

$$
I_{\mathrm{o}}=I-R_{I} E_{(B-V)}
$$

where $R_{I}$ is the coefficient of the total to the differential extinction for the $I$-band. Combining these two equations with the Period-Luminosity-Color relation (PLC relation)

$M_{I}=a \log P+b+c(V-I)_{\mathrm{o}}$

and with the definition of the distance modulus one obtains:

$\langle\mu\rangle=\frac{\left\langle\mu_{V}\right\rangle-\left(R_{V} / R_{I}\right)\left\langle\mu_{I}\right\rangle}{1-\left(R_{V} / R_{I}\right)}$

where,

$$
\begin{aligned}
& \left\langle\mu_{V}\right\rangle=\langle V\rangle-\left(a_{V} \log P+b_{V}\right) \\
& \left\langle\mu_{I}\right\rangle=\langle I\rangle-\left(a_{I} \log P+b_{I}\right) .
\end{aligned}
$$

Let us recall that in these equations $\langle X\rangle$ means average over all the colors at the considered $\log P$. Because all these expressions are linear it is equivalent to make the calculation for each individual Cepheid and to deduce the mean $\langle\mu\rangle$ afterwards. This is the method used in the present paper. It is equivalent to the Wesenheit function method as already emphasized by several authors (e.g., Tanvir 1997).

\subsection{The observational material}

In 1999 we constructed an Extragalactic Cepheid database (Lanoix et al. 1999b). The updated version contains 6685 measurements for 2449 Cepheids in 46 galaxies.
The full contents of the extragalactic part is available in electronic form as described in Paper I.

Let us recall briefly the characteristics of the sample extracted from this database. Each light curve has been inspected. Only light curves classified as "Normal" (see Lanoix et al. 1999b) are used. Only the mean $V$ and $I$ band magnitudes are kept in the present study. When several magnitudes are averaged from different sources, we keep the mean only if the mean error is less than $0.05 \mathrm{mag}$. The final sample results in 1840 extragalactic Cepheids belonging to 36 galaxies. It is also available in electronic form (see Paper I). The source codes of measurements are given for each galaxy in Table 1. The full references appear in the bibliography with their codes.

\subsection{Adopted PL relations}

From HIPPARCOS measurements of 238 galactic Cepheids $^{1}$ we obtained unbiased $V$ - and $I$-band PeriodLuminosity relations (Lanoix et al. 1999) using the treatment described by Feast \& Catchpole (1997):

$\left\langle M_{V}\right\rangle=-2.77 \log P-1.44 \pm 0.05$

$\left\langle M_{I}\right\rangle=-3.05 \log P-1.81 \pm 0.09$.

Let us recall that the slopes of the PL relations could not be determined from HIPPARCOS measurements. Only the zero points (i.e. the mean absolute magnitude at a mean Period) have been fixed. The $V$-band slope $a_{V}=$ -2.77 was adopted from a mean of different values obtained for LMC (Caldwell \& Laney 1991; Madore \& Freedman 1991; Tanvir 1997; Gieren et al. 1998). The $I$-band slope $a_{I}=-3.05$ resulted from $a_{V}$ and from the slope (0.28) of the mean Period-Color relationship. Equation (4) is applied to the 1840 Cepheids of our sample. We adopt the ratio $R_{V} / R_{I}=1.69$ (Cardelli et al. 1989). For each extragalactic Cepheid of each host galaxy we plot the apparent distance modulus vs. $\log P$.

It is important to emphasize that we adopt the LMC slopes and assume that it is universal and bias free. The question of the choice of the slope will be addressed separately.

\subsection{The correction for incompleteness bias}

Sandage (1988) noticed that truncating a complete sample of Cepheids in LMC changes the slope of the resulting PL relation. After Sandage this question remained untouched for several years. Kelson (1996) mentioned the incompleteness bias and suggested one use the inverse slope to correct the effect. Then, the effects of the bias were described from observation (Paturel et al. 1997a) and from simulation (Lanoix et al. 1999a). This effect is not negligible, e.g., it can affect the distance modulus by $0.4 \mathrm{mag}$ for a galaxy like NGC4536. One way to reduce the effect consists in using a magnitude limiting cut-off

\footnotetext{
1174 Cepheids in $I$-band.
} 
Table 1. Sample of extragalactic Cepheids. Column 1: Name of the host galaxy; Col. 2: list of reference codes.

\begin{tabular}{|c|c|}
\hline galaxy & Cepheid reference code \\
\hline IC 1613 & Fr88a Sa88a \\
\hline IC 4182 & Sah94 Gib99 \\
\hline LMCogle & Uda99 \\
\hline NGC 1326A & Pro99 \\
\hline NGC 1365 & Sil98 \\
\hline NGC 1425 & Mou99 \\
\hline NGC 2090 & Phe98 \\
\hline NGC 224 & Fre90 \\
\hline NGC 2541 & Fer98 \\
\hline NGC 300 & Fre92 Wal88 \\
\hline NGC 3031 & Fre94 \\
\hline NGC 3109 & Mus98 Sa88b \\
\hline NGC 3198 & Kel99 \\
\hline NGC 3319 & Sak99 \\
\hline NGC 3351 & Gra97 \\
\hline NGC 3368 & Tan95 Gib99 \\
\hline NGC 3621 & Raw97 \\
\hline NGC 3627 & Sah99 Gib99 \\
\hline NGC 4258 & Mao99 \\
\hline NGC 4321 & Fer96 \\
\hline NGC 4414 & Tur98 \\
\hline NGC 4496A & Sh96c Gib99 \\
\hline NGC 4535 & Mac99 \\
\hline NGC 4536 & Sh96a Gib99 \\
\hline NGC 4548 & Gra99 \\
\hline NGC 4603 & New99 \\
\hline NGC 4639 & Sah97 Gib99 \\
\hline NGC 4725 & Gib98 \\
\hline NGC 5253 & Sah95 Gib99 \\
\hline NGC 5457 & Alv95 Kel96 \\
\hline NGC 598 & Chr87 Fre91 Kin87 Sa88a \\
\hline NGC 6822 & Gal96 Kay67 \\
\hline NGC 7331 & Hug98 \\
\hline NGC 925 & Sil96 \\
\hline SEXA & Pio94 \\
\hline SEXB & Pio94 Sa85b \\
\hline
\end{tabular}

(Freedman et al. 2001). Another way consists of fitting the bias (the variation of the distance modulus with $\log P$ ). A biased distance modulus appears smaller by the quantity (Teerikorpi 1987; see Paper I):

$$
\Delta \mu=-\sigma \sqrt{\frac{2}{\pi}} \frac{e^{-A^{2}}}{1+\operatorname{erf}(A)}
$$

where

$A=\frac{V_{\lim }-\mu-a_{v} \log P-b_{v}}{\sigma \sqrt{2}}$ and

$\operatorname{erf}(x)=\frac{2}{\sqrt{\pi}} \int_{0}^{x} e^{-t^{2}} \mathrm{~d} t$.

Here we assume, consistent with Lanoix et al. (1999), Paper I and Freedman et al. (2001), that the relevant dispersion $\sigma$ is the scatter in the mean absolute magnitude at a fixed $P$. An iterative process is used because the distance modulus must be known to calculate the bias. We start with an initial distance modulus estimated from a simple mean and then, by scanning distance modulus within \pm 1 mag. around the initial value, we search for the best fit. For each galaxy, the limiting magnitude $V_{\lim }$ is taken from Paper I; The initial value of the dispersion $(\sigma)$ is calculated from the uncorrected distance moduli; Then it is adjusted at each step of the iterative process.

For each of the 36 host galaxies we plot the apparent distance moduli given through Eq. (4) as a function of $\log P$ and we superimposed the bias curve obtained after the last iteration. This result appears in Fig. 1 and Table 4. The standard error on the distance moduli is an internal error.

We want now to see how the results are modified when different PL relations are used. We want also to test the stability of the solution.

\section{Discussion}

\subsection{Comparison with an independent treatment}

Freedman et al. (2001) recently published their HST keyproject distance moduli (The Hubble Space Telescope Key Project on the Extragalactic Distance Scale) calibrated with LMC, assuming $\mu(\mathrm{LMC})=18.5$. The agreement between HSTKP distance moduli and those calculated here is good for the 31 galaxies in common. We do not confirm the tendency found in Paper I that their distance moduli are smaller than ours above $\mu=30$. A direct regression of our distance moduli vs. HSTKP distance moduli $\mu$ (HSTKP) (uncorrected for metallicity effect, denoted $\mu_{\mathrm{o}}$ and presented in Col. 8 of Table 4 in the paper by Freedman et al.) leads to a slope which is not significantly different from one $(1.012 \pm 0.005)$ and a zero point difference which is not significantly different from zero $(-0.027 \pm 0.016)$ at the 0.01 probability level (the Student's t-test requires $\left.t_{0.01}(\nu=30)>2.75\right)$. The standard deviation is $\sigma=0.090$. Thus, assuming both determinations carry the same uncertainty, this means that our distances are good within $0.09 / \sqrt{2}=0.06 \mathrm{mag}$. This is not perfectly exact because both solutions are not fully independent (except for the zero-point calibration). In particular, we use the same ratio $R_{V} / R_{I}=1.69$ (Cardelli et al. 1989) and most of the observations are the same.

\subsection{Influence of the choice of the PL relations}

The agreement between our solution and the HSTKP one may appear strange because our relations 7 and 8 
G. Paturel et al.: Calibration of the distance scale. II.
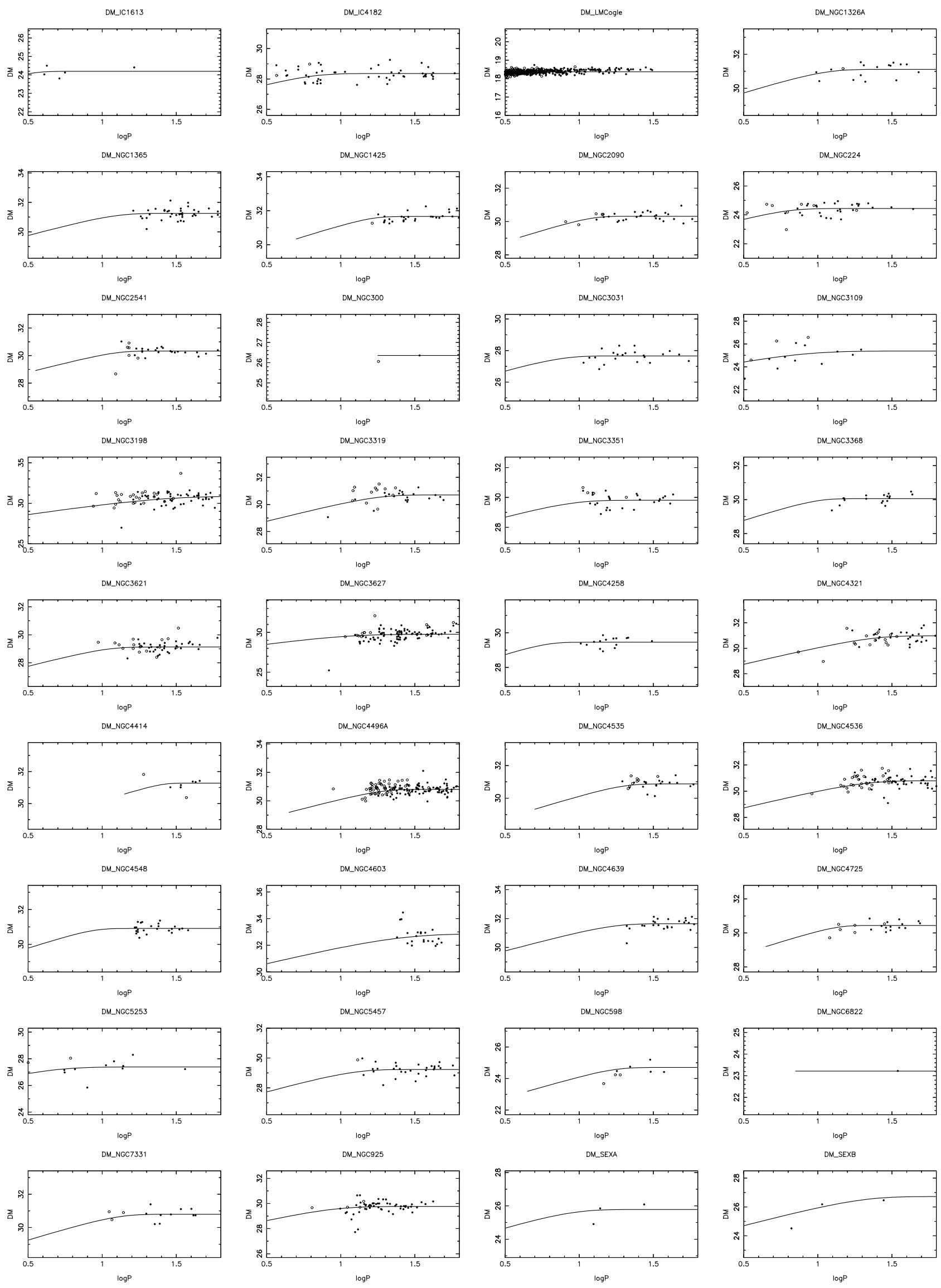

Fig. 1. Distance moduli ( $y$-axis) from the PL relations vs. $\log P(x$-axis) for each host galaxy. 
do not differ very much from the old calibration ${ }^{2}$ (Madore \& Freedman 1991) which was revised (Freedman et al. 2001) using Udalski et al. (1999) results on LMC. The new HSTKP calibration is:

$$
\begin{aligned}
& \left\langle M_{V}\right\rangle=-2.76 \log P-1.45 \\
& \left\langle M_{I}\right\rangle=-2.96 \log P-1.94 .
\end{aligned}
$$

Thus, it will be interesting to apply these new relations to our own sample to see if the agreement is not fortuitously due to the data. We will keep $R_{V} / R_{I}=1.69$. This is the value adopted in the HSTKP, in our Paper I and in the present paper. In Table 2 we give the mean shift $\langle\Delta \mu\rangle=$ $\langle\mu\rangle-\langle\mu(H S T K P)\rangle$ for different solutions applied to our data. The result is also shown in Figs. 2a-d The definitions of the different solutions are the following:

Solution \#1: HIPPARCOS: The PL relations are Rel. 7 and 8.

Solution \#2: HSTKP-PL: The PL relations are those adopted by Freedman et al. (2001), i.e., Rel. 14 and 15 .

Solution \#3: GFG-SOSIE: This is the solution from Paper I. Slopes and zero-points are not required explicitly. The calibration is based on the GFG sample.

Solution \#4: test: This is a test of a change of slopes (the zero-points being recalculated from the HIPPARCOS calibration as described in footnote 3 ). This solution is discussed below.

The distance moduli found with the HSTKP-PL relations are similar to the final ones published by the HSTKP team (see Fig. 2b) but the difference $\langle\Delta \mu\rangle=0.045 \pm 0.015$ is significant at the $3-\sigma$ level. This difference can be explained by the fact that the data are not exactly the same and that the correction for the incompleteness bias is made in a different way. Part of the difference can be explained as a consequence of the fact that changes in the photometric zero point adopted by the HSTKP (Stetson 1998) have not been reflected in the Lanoix et al. compilation which is used in this paper. The Lanoix compilation uses slightly different zero points for different galaxies. For $50 \%$ of the galaxies of the present sample, the distance moduli are $0.06 \mathrm{mag}$ larger than the distance moduli used by Freedman et al. 2001. The mean observed departure (0.045), although significant, is relatively small in comparison with the departure that could be due to an uncertainty on the PL slope, as illustrated by Figs. 2a-d and Table 2.

The LMC distance modulus is retrieved at $\mu(\mathrm{LMC})=$ 18.5 as assumed for the HSTKP calibration. In fact the difference between the adopted PL relations of solutions 1

\footnotetext{
2 The old Madore \& Freedman (1991) PL relations were:
}

$$
\begin{aligned}
& \left\langle M_{V}\right\rangle=-2.76 \log P-1.40 \\
& \left\langle M_{I}\right\rangle=-3.06 \log P-1.81
\end{aligned}
$$

and 2 is smaller than it appears. Indeed, if one forces a slope of -2.76 in $V$-band (respectively, -2.96 in $I$-band) on the HIPPARCOS zero-point which was obtained at a mean $\log P=0.82$ (see Lanoix et al. 1999) one obtains the corrected PL relations ${ }^{3}$ :

$$
\begin{aligned}
& \left\langle M_{V}\right\rangle=-2.76 \log P-1.45 \\
& \left\langle M_{I}\right\rangle=-2.96 \log P-1.88 .
\end{aligned}
$$

These corrected PL relations do not differ very much from the new HSTKP PL ones. This explains the relatively good agreement between solution 1 and the HSTKP results (or, equivalently, with solution \#2).

On the contrary, the last solution (GFG-SOSIE) shows a departure from the first two solutions (HIPPARCOS and HSTKP-PL) especially above $10 \mathrm{Mpc}(\mu=30)$. It seems that one retrieves the dilemma emphasized in Paper I that either the HSTKP distance moduli may have a small residual bias or that the GFG sample may overestimate the absolute magnitude for long periods.

If the PL slopes are changed into -3.0 for the $V$-band (respectively, -3.3 for the $I$-band) as suggested by the results of GFG or Laney \& Stobie (1994), the zero-points being still recalculated from our HIPPARCOS calibration, then the results (solution \#4 in Table 2) are compatible with those of our solution \#3 (the mean shift $\Delta \mu=+0.163$ while the solution \#3 gives $\Delta \mu=+0.161$ ). Thus, we suspect that the PL slopes adopted from LMC are not valid for all kinds of galaxies. This question will be discussed elsewhere. Here, we will adopt our solution \#1. The uncertainty due to the slope will be discussed in the error budget.

\subsection{Influence of the incompleteness bias}

As we explained above, the determination of $V_{\lim }$ may affect the correction of the incompleteness bias. In order to evaluate the mean effect we repeated the calculation of distance moduli varying $V_{\lim }$ over the range $\left(V_{\lim }-0.5, V_{\lim }+0.5\right)$. The mean changes of distance moduli, $\Delta \mu^{-}$and $\Delta \mu^{+}$respectively, are given in Table 3 . The mean change is less than 0.05 mag when $V_{\lim }$ changes by $0.5 \mathrm{mag}$. Nevertheless, individual changes may be larger than this mean value. Hence, for each galaxy we give the individual $\Delta \mu^{-}$and $\Delta \mu^{+}$in Table 4 . The change is generally smaller than 0.1 mag. Because it depends on each individual galaxy, it introduces a random error. Assuming that the uncertainty on the limiting magnitude is \pm 0.5 , the resulting error will be calculated for each galaxy from the relation ${ }^{4} \sigma_{\text {bias }}=1.25|\Delta \mu|$. For $\Delta \mu$ we will adopt the maximum between $\Delta \mu^{+}$and $\Delta \mu^{-}$. It will be taken into account in the error budget.

\footnotetext{
${ }^{3}$ With obvious notation the new zero-point is $b^{\prime}=b+\left(a-a^{\prime}\right)\langle\log P\rangle$.

${ }^{4}$ For a Gaussian distribution $\mathcal{G}(\bar{x}, \sigma)$, the standard deviation is $\sigma=1.25\langle|\Delta x|\rangle$.
} 
Table 2. Distance moduli calculated from different solutions (see text) with $R_{V} / R_{I}=1.69$.

\begin{tabular}{llllll}
\hline \hline Solution & $a_{V}$ & $b_{V}$ & $a_{I}$ & $b_{I}$ & $\langle\Delta \mu\rangle=\langle\mu-\mu($ HSTKP $)\rangle$ \\
\hline \#1: HIPPARCOS & -2.77 & -1.44 & -3.05 & -1.81 & $0.027 \pm 0.016$ \\
\#2: HSTKP-PL & -2.760 & -1.458 & -2.962 & -1.942 & $0.045 \pm 0.015$ \\
\#3: GFG-SOSIE ${ }^{a}$ & \multicolumn{2}{c}{ slopes and ZP not required } & & $0.161 \pm 0.029$ \\
\#4: test & -3.0 & -1.02 & -3.3 & -1.44 & $0.163 \pm 0.026$ \\
\hline
\end{tabular}

${ }^{a}$ Based on the GFG sample.

Table 3. Test of the stability of the results. We give the departure from our reference solution for several $R_{V} / R_{I}$ ratios.

\begin{tabular}{rrr}
\hline \hline$\Delta V_{\lim }$ & $R_{V} / R_{I}$ & $\mu-\mu_{\text {ref }}$ \\
\hline 0.0 & 1.89 & $-0.05 \pm 0.04$ \\
0.0 & 1.79 & $-0.03 \pm 0.01$ \\
0.0 & 1.69 & 0 \\
0.0 & 1.59 & $+0.04 \pm 0.03$ \\
0.0 & 1.49 & $+0.09 \pm 0.06$ \\
\hline-0.50 & 1.69 & $-0.04 \pm 0.14$ \\
-0.25 & 1.69 & $-0.02 \pm 0.09$ \\
+0.25 & 1.69 & $+0.02 \pm 0.04$ \\
+0.50 & 1.69 & $+0.03 \pm 0.07$ \\
\hline
\end{tabular}

\subsection{Influence of the ratio $R_{V} / R_{I}$}

In order to check the stability of our adopted solution, we repeated the calculations with a variation of the ratio $R_{V} / R_{I}$ by \pm 0.2 (about $10 \%$ ). The results are summarized in Table 3, where we give the difference between the mean distance moduli obtained with different $R_{V} / R_{I}$ ratios and our reference solution based on $R_{V} / R_{I}=1.69$. A change of $R_{V} / R_{I}$ by $\pm 10 \%$ changes the distance moduli by less than $0.1 \mathrm{mag}$. If the $R_{V} / R_{I}$ is not the same for all galaxies, this introduces a dispersion of the calculated distance moduli, but not a systematic effect. Assuming that the uncertainty on $R_{V} / R_{I}$ is \pm 0.2 , the resulting error is about $\sigma_{\text {extinction }}=1.25|\Delta \mu| \approx 0.09$. It will be taken into account in the error budget.

\subsection{Influence of metallicity}

The problem of metallicity was first recognized by Iben (1967). According to Freedman \& Madore (1990) the coefficients of the PL relation are slightly dependent on metallicity. Thus, the zero-point of the extragalactic distance scale would be slightly dependent on the metallicity. However, it has been argued that the correction of interstellar absorption is particularly sensitive to the metallicity (Beaulieu et al. 1997).

Most empirical investigations (Gould et al. 1994; Sasselov et al. 1997; Kennicut et al. 1998) find a positive effect ranging from 0.24 (Kennicut et al. 1998) to 0.56 (Gould et al. 1994). More recently, Udalski (1999) confirmed Freedman \& Madore (1990) result that the metallicity effect is negligible. In the theoretical approaches, the results of linear computations (Chiosi et al. 1993; Sandage et al. 1999; Alibert et al. 1999) suggest a small negative effect. However, using non-linear models (Bono et al. 2000) Caputo et al. (2000) found a positive effect.

Owing to these puzzling results, we do not expect to solve the problem in the classical way. Instead, we will avoid it by considering that the method is valid only for galaxies with nearly the same metallicity as the calibrating Cepheids (i.e., nearly Solar metallicity). Indeed, there is a clear correlation (Fig. 3) between the morphological type code of the host galaxy and its metallicity $12+\log \mathrm{O} / \mathrm{H}$ as listed by Caputo et al. (2000). Thus, we will consider that only spiral galaxies over the range Sa-Scd (i.e., type codes 1-7) should be considered as reliable. When this restriction is applied, we may consider that the uncertainty due to metallicity is negligibly small.

\subsection{Error budget}

In Table 4 we summarize our determinations of distance moduli for 36 galaxies calibrated with galactic Cepheids. The provisional distance moduli are calculated from a weighted mean of our two determinations (GFG-SOSIE and HIPPARCOS). The weight is the inverse of the square of the individual mean error. The final error on the mean distance modulus is the "actual error" (Paturel et al. 1997) which takes into account the individual errors (uncertainty due to the data) and the discrepancy between the solutions (uncertainty due to the adopted zero-point). This uncertainty will be designated $\sigma_{\text {zero-point }}$. The uncertainties due to the incompleteness bias correction and to the extinction correction are added. The uncertainty resulting from a possible metallicity effect will be neglected but galaxies with a morphological type out of the accepted range (Sa-Scd) are given in parenthesis.

We believe that another source of uncertainty can result from the choice of the slope. This is partially taken into account in $\sigma_{\text {zero-point }}$ because the GFG-SOSIE solution does not require knowledge of the slope. This additional uncertainty is $1.25|\Delta \mu| / \sqrt{2}$ (i.e. $\approx 0.1 \mathrm{mag}$ with $\Delta \mu \approx 0.11)$.

Finally, the total uncertainty $\sigma_{\text {total }}$ (internal plus external) is calculated for each individual galaxy from:

$\sigma_{\text {total }}^{2}=\sigma_{\text {zero-point }}^{2}+\sigma_{\text {bias }}^{2}+\sigma_{\text {extinction }}^{2}+\sigma_{\text {slope }}^{2}$.

This estimate is given with the provisional distance modulus in Table 4. 

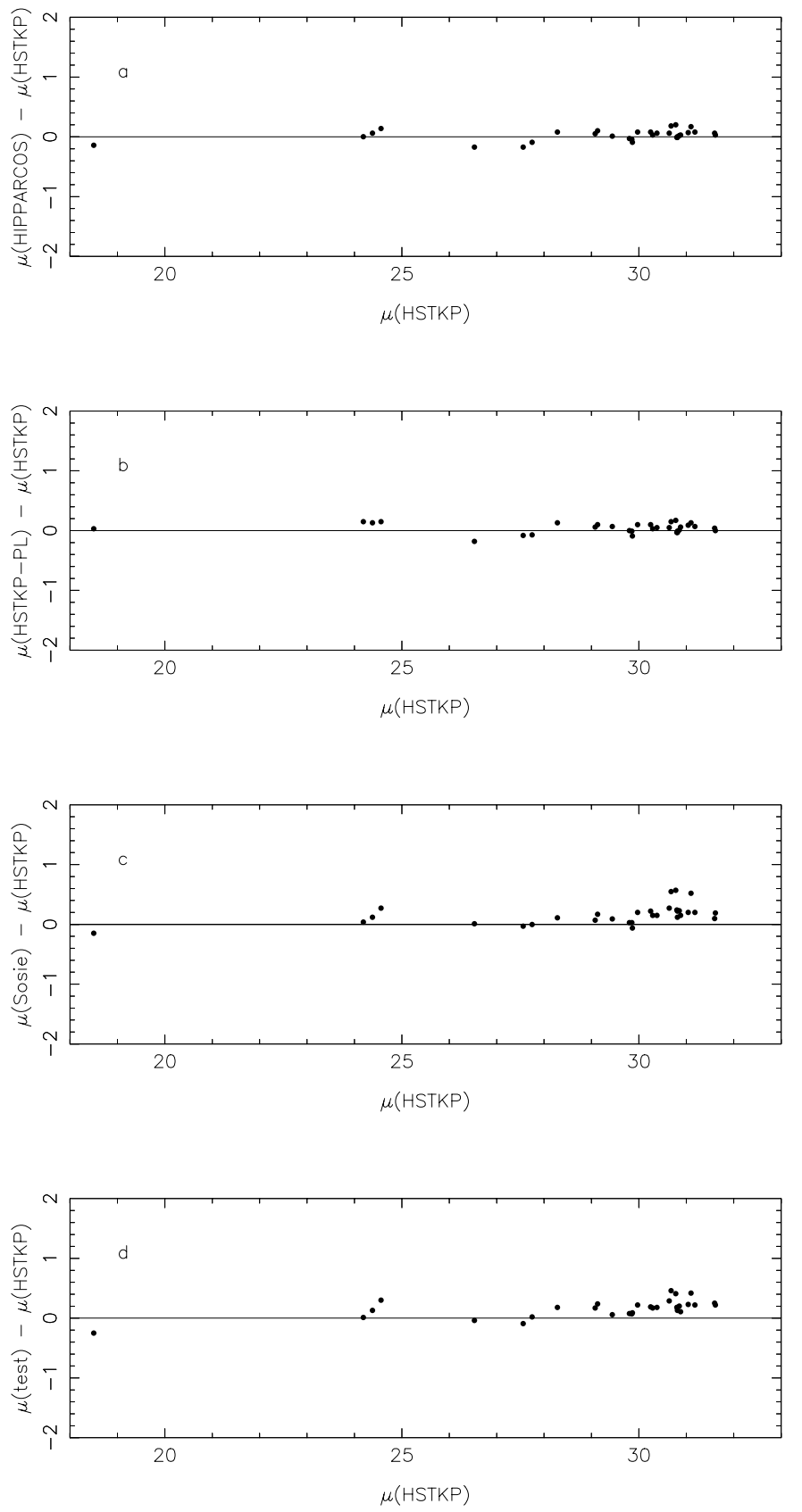

Fig. 2. Comparison between the distance moduli from different solutions (Table 2) and the HSTKP distance moduli $\mu_{\mathrm{o}}$. The different solutions are applied on the same data. a)The first solution (HIPPARCOS calibration, this paper) is in reasonnably good agreement with the HSTKP solution. b) The second solution (HSTKP-PL relations applied on the present data). c) The third solution (SOSIE method applied on the same data, Paper I) shows a departure from the HSTKP solution, especially at large distance $(\mu>30)$. d) The fourth solution (test solution with larger PL slopes) shows the same trend as the third one and suggests that the choice of the PL slopes may have an important effect, especially at large distances.

A comparison with the HSTKP distance moduli is presented in Fig. 4. Excepting two galaxies at large distances (NGC 4321 and NGC 3198 noted with a (:) in Table 4), the agreement is good.

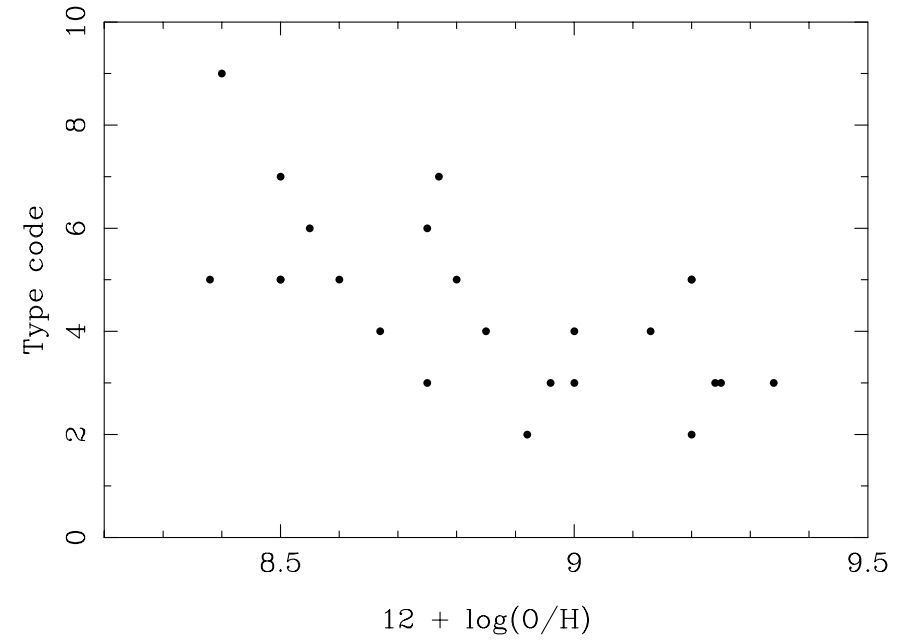

Fig. 3. Correlation between the morphological type code of the host galaxy and its metallicity $12+\log \mathrm{O} / \mathrm{H}$.

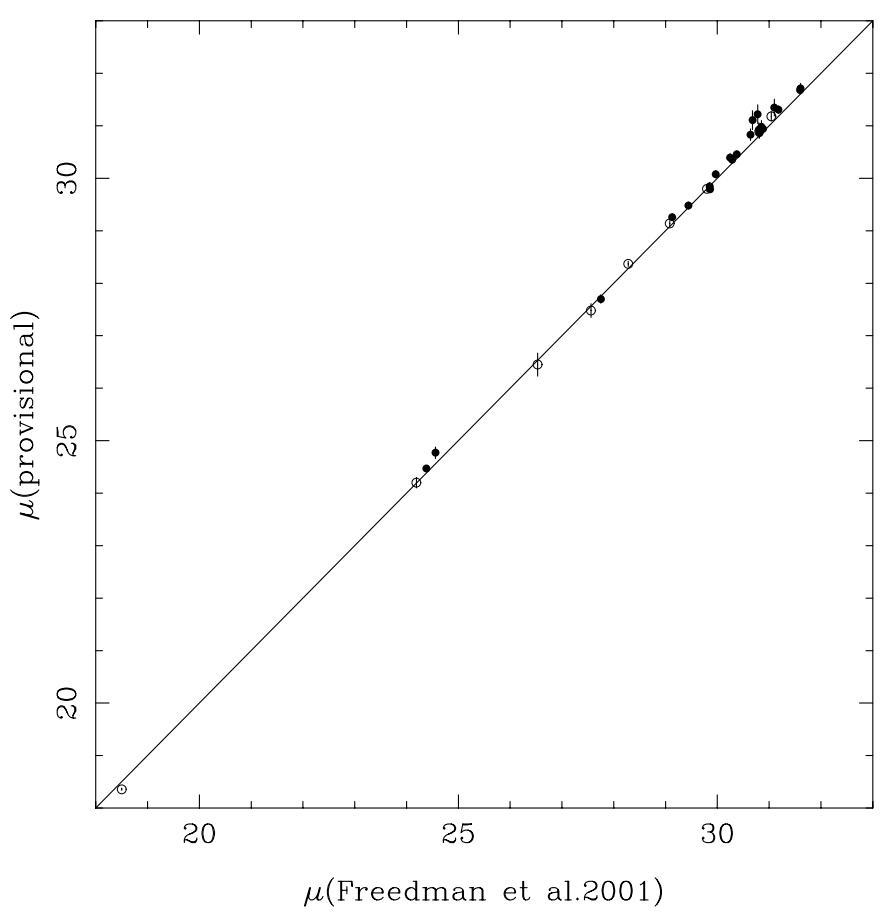

Fig. 4. Comparison of the distance moduli from Freedman et al. (2001) and from this paper. The general agreement is satisfactory despite the being calibration is independent. Late type galaxies are represented with open circles.

\section{Conclusion}

The preliminary distance moduli obtained in the first two papers of this series were analyzed to search for possible residual bias. Distance moduli from Paper I and from this paper agree reasonably well within $0.1 \mathrm{mag}$, although they are based on two independent calibration (GFG-SOSIE and HIPPARCOS) and two independent methods (Sosie and classical PL relations).

The discussion of the stability of the solution shows that the slope of the PL relations is still under question. The LMC slope in $V$-band (and maybe for all late type 
Table 4. Distance moduli. Column 1: Name of the host galaxy. Column 2: Morphological type code (de Vaucouleurs et al. 1991). Columns 3: Distance modulus from Paper I (calibration with galactic Cepheids from GFG sample) with its internal error. Column 4: The distance modulus from the present paper (calibration with galactic Cepheids from HIPPARCOS) with its internal error. Columns 5, 6: changes of distance modulus when the limiting magnitude is changed by \pm 0.5 mag. Column 7 : The distance modulus from HSTKP with its random uncertainty (Cols. 8 and 9 in Freedman et al. 2001). Column 8: Adopted distance modulus with its total error. Distance moduli given in parenthesis correspond to late type galaxies.

\begin{tabular}{|c|c|c|c|c|c|c|c|}
\hline galaxy & Type & GFG-SOSIE & HIPPARCOS & $\Delta \mu^{-}$ & $\overline{\Delta \Delta \mu^{+}}$ & HSTKP & Provisional $\mu$ \\
\hline IC 1613 & 10 & $24.23 \pm 0.19$ & $24.19 \pm 0.11$ & 0.09 & -0.02 & $24.19 \pm 0.15$ & $(24.20 \pm 0.20)$ \\
\hline IC 4182 & 9 & $28.39 \pm 0.07$ & $28.36 \pm 0.06$ & -0.02 & -0.03 & $28.28 \pm 0.06$ & $(28.37 \pm 0.15)$ \\
\hline LMCogle & 9 & $18.35 \pm 0.03$ & $18.37 \pm 0.00$ & 0.01 & 0.00 & 18.50 & $(18.37 \pm 0.14)$ \\
\hline NGC $1326 \mathrm{~A}$ & 9 & $31.24 \pm 0.09$ & $31.11 \pm 0.10$ & 0.05 & -0.04 & $31.04 \pm 0.10$ & $(31.18 \pm 0.17)$ \\
\hline NGC 1365 & 3 & $31.38 \pm 0.07$ & $31.26 \pm 0.06$ & 0.00 & -0.01 & $31.18 \pm 0.05$ & $31.31 \pm 0.15$ \\
\hline NGC 1425 & 3 & $31.70 \pm 0.06$ & $31.66 \pm 0.05$ & 0.07 & -0.05 & $31.60 \pm 0.05$ & $31.68 \pm 0.17$ \\
\hline NGC 2090 & 5 & $30.44 \pm 0.07$ & $30.32 \pm 0.05$ & 0.02 & 0.00 & $30.29 \pm 0.04$ & $30.36 \pm 0.15$ \\
\hline NGC 224 & 3 & $24.50 \pm 0.08$ & $24.44 \pm 0.07$ & 0.14 & -0.04 & $24.38 \pm 0.05$ & $24.47 \pm 0.23$ \\
\hline NGC 2541 & 5 & $30.47 \pm 0.07$ & $30.33 \pm 0.06$ & -0.02 & -0.03 & $30.25 \pm 0.05$ & $30.39 \pm 0.16$ \\
\hline NGC 300 & 6 & $26.54 \pm 0.29$ & $26.36 \pm 0.29$ & 0.00 & -0.15 & $26.53 \pm 0.07$ & $26.45 \pm 0.32$ \\
\hline NGC 3031 & 2 & $27.75 \pm 0.10$ & $27.66 \pm 0.08$ & 0.03 & -0.02 & $27.75 \pm 0.08$ & $27.70 \pm 0.16$ \\
\hline NGC 3109 & 9 & $25.10 \pm 0.16$ & $25.38 \pm 0.22$ & -0.08 & 0.16 & & $(25.20 \pm 0.31)$ \\
\hline NGC 3198 & 5 & $31.23 \pm 0.07$ & $30.86 \pm 0.10$ & 0.05 & -0.02 & $30.68 \pm 0.08$ & $31.11: \pm 0.23$ \\
\hline NGC 3319 & 5 & $30.91 \pm 0.06$ & $30.70 \pm 0.08$ & 0.03 & 0.02 & $30.64 \pm 0.09$ & $30.83 \pm 0.18$ \\
\hline NGC 3351 & 3 & $29.88 \pm 0.08$ & $29.81 \pm 0.09$ & 0.04 & 0.04 & $29.85 \pm 0.09$ & $29.85 \pm 0.16$ \\
\hline NGC 3368 & 2 & $30.17 \pm 0.10$ & $30.05 \pm 0.06$ & 0.06 & -0.02 & $29.97 \pm 0.06$ & $30.08 \pm 0.17$ \\
\hline NGC 3621 & 6 & $29.15 \pm 0.07$ & $29.13 \pm 0.06$ & 0.02 & 0.03 & $29.08 \pm 0.06$ & $29.14 \pm 0.15$ \\
\hline NGC 3627 & 3 & $29.80 \pm 0.06$ & $29.77 \pm 0.07$ & 0.03 & -0.02 & $29.86 \pm 0.08$ & $29.79 \pm 0.15$ \\
\hline NGC 4258 & 4 & $29.53 \pm 0.10$ & $29.45 \pm 0.07$ & 0.05 & 0.00 & $29.44 \pm 0.07$ & $29.48 \pm 0.16$ \\
\hline NGC 4321 & 4 & $31.35 \pm 0.06$ & $30.98 \pm 0.08$ & 0.00 & -0.06 & $30.78 \pm 0.07$ & $31.22: \pm 0.24$ \\
\hline NGC 4414 & 5 & $31.62 \pm 0.09$ & $31.27 \pm 0.05$ & 0.13 & -0.06 & $31.10 \pm 0.05$ & $31.35 \pm 0.26$ \\
\hline NGC 4496A & 7 & $31.03 \pm 0.04$ & $30.81 \pm 0.03$ & 0.01 & 0.03 & $30.81 \pm 0.03$ & $30.89 \pm 0.18$ \\
\hline NGC 4535 & 5 & $31.08 \pm 0.07$ & $30.87 \pm 0.07$ & -0.07 & 0.03 & $30.85 \pm 0.05$ & $30.98 \pm 0.20$ \\
\hline NGC 4536 & 4 & $31.04 \pm 0.06$ & $30.79 \pm 0.07$ & -0.02 & 0.00 & $30.80 \pm 0.04$ & $30.93 \pm 0.19$ \\
\hline NGC 4548 & 3 & $31.03 \pm 0.08$ & $30.91 \pm 0.05$ & 0.06 & 0.00 & $30.88 \pm 0.05$ & $30.94 \pm 0.17$ \\
\hline NGC 4603 & 5 & $33.70 \pm 0.09$ & $32.86 \pm 0.15$ & 0.61 & -0.11 & & $33.48 \pm 0.86$ \\
\hline NGC 4639 & 4 & $31.80 \pm 0.08$ & $31.64 \pm 0.07$ & 0.10 & 0.00 & $31.61 \pm 0.08$ & $31.71 \pm 0.21$ \\
\hline NGC 4725 & 2 & $30.53 \pm 0.11$ & $30.44 \pm 0.06$ & -0.08 & -0.05 & $30.38 \pm 0.06$ & $30.46 \pm 0.18$ \\
\hline NGC 5253 & $6 ?$ & $27.53 \pm 0.14$ & $27.39 \pm 0.18$ & 0.23 & 0.00 & $27.56 \pm 0.14$ & $27.48 \pm 0.34$ \\
\hline NGC 5457 & 5 & $29.30 \pm 0.07$ & $29.23 \pm 0.07$ & 0.03 & 0.01 & $29.13 \pm 0.11$ & $29.26 \pm 0.15$ \\
\hline NGC 598 & 5 & $24.83 \pm 0.12$ & $24.70 \pm 0.13$ & -0.28 & -0.22 & $24.56 \pm 0.10$ & $24.77 \pm 0.39$ \\
\hline NGC 6822 & 10 & $23.38 \pm 0.52$ & $23.22 \pm 0.52$ & 0.00 & 0.00 & & $(23.30 \pm 0.40)$ \\
\hline NGC 7331 & 5 & $30.93 \pm 0.12$ & $30.80 \pm 0.11$ & 0.12 & 0.00 & $30.81 \pm 0.09$ & $30.86 \pm 0.22$ \\
\hline NGC 925 & 6 & $29.83 \pm 0.06$ & $29.77 \pm 0.07$ & 0.00 & -0.05 & $29.80 \pm 0.04$ & $29.80 \pm 0.16$ \\
\hline SEXA & 10 & $25.75 \pm 0.23$ & $25.78 \pm 0.26$ & 0.31 & -0.13 & & $(25.76 \pm 0.44)$ \\
\hline SEXB & 10 & $26.77 \pm 0.18$ & $26.72 \pm 0.30$ & -0.18 & -0.22 & & $(26.76 \pm 0.35)$ \\
\hline
\end{tabular}

galaxies) seems well fixed $\left(a_{V} \approx-2.77\right)$ but several studies (GFG, LS), including this paper, show that the slope for galactic Cepheids (and maybe for all Sa-Scd galaxies) could be steeper $\left(a_{V} \approx-3\right)$. If one adopts a slope $a_{V}=-2.76$ the results are in good agreement with the results of HSTKP. If one adopts a slope $a_{V}=-3.0$ the distance moduli must be increased, on average, by 0.1 mag.

When all sources of errors are taken into account, the mean standard deviation of the final distance modulus is about 0.20 mag.

The correlation between metallicity and morphological type of hosts galaxies suggests to limit the validity of our distances to spiral galaxies (Sa-Scd) that have the same metallicity as our calibration sample.

For NGC 4258 our distance modulus, $\mu=29.48 \pm 0.16$, is compatible with the maser determination $\mu=29.28 \pm$ 0.15 (Herrnstein et al. 1999) and it is in good agreement with the revised distance modulus $\mu=29.48 \pm 0.15$ (Newman et al. 2001).

If it is confirmed that the slopes of PL relations have to be adapted to the morphological type of each host galaxy, these distance moduli could be modified. Further, using the local Hubble flow for providing independent reference distances, an additional analysis of the bias on primary 
calibration (Teerikorpi \& Paturel 2002) suggests the existence of another bias. The problem of distance calibration is not yet resolved.

Acknowledgements. We thank the HST teams for making their data available in the literature prior to the end of the project. We thank R. Garnier, J. Rousseau and P. Lanoix for having participated in the maintenance of our Cepheid database. P.T. acknoledges the support by the Academy of Finland (project "Cosmology from the local to the deep galaxy universe". We thank the anonymous referee for valuable remarks.

\section{References}

Alibert, Y., Baraffe, I., Hauschildt, P., \& Allard, F. 1999, A\&A, 344,551

Alves, D. R., \& Cook, K. H. 1995, AJ, 110, 192 (Alv95)

Beaulieu, J. P., Sasselov, D. D., Renault, C., et al. 1997, A\&A, 318, L47

Bono, G., Castellani, V., \& Marconi, M. 2000, ApJ, 529, 293

Caldwell, J. A. R., \& Coulson, I. M. 1987, AJ, 93, 1090

Caldwell, J. A. R., Laney, C. D. 1991, in The Magellanic Clouds, ed. R. Haynes, \& D. Milne (Kluwer, Dordrecht), Proc. IAU Symp., 148, 249

Caputo, F., Marconi, M., Musella, I., \& Santolamazza, P. 2000, A\&A, 359, 1059

Cardelli, J. A., Clayton, G. C., \& Mathis, J. S. 1989, ApJ, 345, 245

Chiosi, C., Wood, P. R., \& Capitanio, N. 1993, ApJS, 86, 541 Christian, C. A., \& Schommer, R. A. 1987, AJ, 93, 557 (Chr87)

Feast, M. W., \& Catchpole, R. M. 1997, MNRAS, 286, L1

Ferrarese, L., Freedman, W. L., Hill, R. J., et al. 1996, ApJ, 464, 568 (Fer96)

Ferrarese, L., Bresolin, F., Kennicutt, R. C., Jr., et al. 1998, ApJ, 507, 655 (Fer98)

Freedman, W. L., Hughes, S. M., Madore, B.F., et al. 1994, ApJ, 427, 628 (Fre94)

Freedman, W. L. 1988, ApJ, 326, 691 (Fr88a)

Freedman, W. L., \& Madore, B. F. 1990, ApJ, 365, 186 (Fre90)

Freedman, W. L., Madore, B. F., Gibson, B. K., et al. 2001, ApJ, 553, 47 (HSTKP)

Freedman, W. L., Madore, B. F., Hawley, S. L., et al. 1992, ApJ, 396, 80 (Fre92)

Freedman, W. L., Wilson, C. D., \& Madore, B. F. 1991, ApJ, 372, 455 (Fre91)

Gallart, C., Aparicio, A., \& Vichez, J. M. 1996, AJ, 112, 1928 (Gal96)

Gibson, B. K., et al. 1998 [astro/ph981003], unpublished (Gib98)

Gibson, B. K., Hughes, S. M. G., Stetson, P. B., et al. 1999, ApJ, 512, 48 (Gib99)

Gieren, W., Fouqué, P., \& Gomez, M. 1998, ApJ, 496, 17 (GFG)

Graham, J. A., Phelps, R. L., Freedman, W. L., et al. 1997, ApJ, 477, 535 (Gra97)

Graham, J. A., Ferrarese, L., Freedman, W. L., et al. 1999, ApJ, 516, 626 (Gra99)

Herrnstein, J. R., Moran, J. M., Greenhill, L. J., et al. 1999, Nature, 400, 539

Hughes, S. M. G., Han, M. , Hoessel, J., et al. 1998, ApJ, 501, 32 (Hug98)
Iben, I. 1967, ARA\&A, 5, 606

Kayser, S. E. 1967, AJ, 72, 134 (Kay67)

Kelson, D. D., Illingworth, G. D., Freedman, W. F., et al. 1996, ApJ, 463, 26 (Kel96)

Kelson, D. D., Illingworth, G. D., Saha, A., et al. 1999, ApJ, 514, 614 (Kel99)

Kennicut, R. C., Stetson, P. B., Saha, A., et al. 1998, ApJ, 498, 181

Kinman, T. D., Mould, J. R., \& Wood, P. R. 1987, AJ, 93, 833 (Kin87)

Laney, C. D., \& Stobie, R. S. 1994, MNRAS, 266, 441 (LS)

Lanoix, P., Paturel, G., \& Garnier, R. 1999, MNRAS, 308, 969

Lanoix, P., Paturel, G., Garnier, R., et al. 1999a, ApJ, 517, 188

Lanoix, P., Paturel, G., Garnier, R., et al. 1999b, Astron. Nach., 320, 1

Lutz, T. E., \& Kelker, D. H. 1973, PASP, 85, 573

Macri, L. M., Huchra, J. P., Stetson, P. B., et al. 1999, ApJ, 521, 155 (Mac99)

Madore, B. F., \& Freedman, W. L. 1991, PASP, 103, 933

Maoz, E., Newman, J. A., Ferrarese, L., et al. 1999, Nature, 401, 351 (Mao99)

Mould, J. R., Hughes, S. M. G., Stetson, P. B., et al. 2000, ApJ, 528, 655 (Mou99)

Musella, I., Piotto, G., \& Capaccioli, M. 1997, AJ, 114, 976 (Mus98)

Newman, J. A., Ferrarese, L., Stetson, P. B., et al. 2001, ApJ, 553,562

Newman, J. A., Zepf, E., Davis, M., et al. 1999, ApJ, 523, 506 (New99)

Paturel, G., Andernach, H., Bottinelli, L., et al. 1997, A\&AS, 124,109

Paturel, G., Lanoix, P., Garnier, R., et al. 1997a, in Proc. of the ESA Symp., Hipparcos Venice '97, ed. M. A. C. Perryman, \& P. L. Bernacca

Paturel, G., Theureau, G., Fouqué, P., et al. 2002, A\&A, 383, 398 (Paper I)

Perryman, M. A. C., Hog, E., Kovalevsky, J., Lindengren, L., \& Turon, C. 1997, in The Hipparcos and Tycho Catalogues, European Space Agency, SP-1200

Phelps, R. L., Sakai, S., Freedman, W. L., et al. 1998, ApJ, 500, 763 (Phe98)

Piotto, G., Capaccioli, M., \& Pellegrini, C. 1994, A\&A, 287, 371 (Pio94)

Pont, F., Charbonnel, C., Lebreton, Y., Mayor, M., \& Turon, C. 1997, ESA Symp., Hipparcos - Venice'97, ESA SP-402 (ESA Noordwijk), 699

Prosser, C. F., Kennicutt, R. C., Jr., Bresolin, F., et al. 1999, ApJ, 525, 80 (Pro99)

Rawson, D. M., Macri, L. M., Mould, J. R., et al. 1997, ApJ, 490, 517 (Raw97)

Saha, A., Labhardt, L., Schwengeler, H., et al. 1994, ApJ, 425, 14 (Sah94)

Saha, A., Sandage, A., Labhardt, L., et al. 1995, ApJ, 438, 8 (Sah95)

Saha, A., Sandage, A., Labhardt, L., et al. 1996, ApJ, 466, 55 (Sh96a)

Saha, A., Sandage, A., Labhardt, L., et al. 1996, ApJS, 107, 693 (Sh96c)

Saha, A., Sandage, A., Tammann, G. A., et al. 1999, ApJ, 522, 802 (Sah99)

Saha, A., Sandage, A., Labhardt, L., et al. 1997, ApJ, 486, 1 (Sah97) 
Sakai, S., Ferrarese, L., Kennicutt, R. C. et al. 1999, ApJ, 523, 540 (Sak99)

Sandage, A. 1988, PASP, 100, 935 (Sa88a)

Sandage, A., \& Carlson, G. 1985b, AJ, 90, 1019 (Sa85b)

Sandage, A., \& Carlson, G. 1988, AJ, 96, 1599 (Sa88b)

Sandage, A., Bell, R. A., \& Tripicco, M. J. 1999, ApJ, 522, 250

Sasselov, D., Beaulieu, J. P., Renault, C., et al. 1997, A\&A, 324,471

Silbermann, N. A., Harding, P., Madore, B. F., et al. 1996, ApJ, 470, 1 (Sil96)

Silbermann, N. A., Harding, P., Ferrarese, L., et al. 1999, ApJ, 515, 1 (Sil98)

Stetson, P. B. 1998, PASP, 110, 1448
Tanvir, N. R., Shanks, T., Ferguson, H. C., et al. 1995, Nature, 337, 27 (Tan95)

Tanvir, N. 1997, in The Extragalactic Distance Scale (Cambridge Univ. Press), ed. M. Livio, 91

Teerikorpi, P. 1987, A\&A, 173, 39

Teerikorpi, P., \& Paturel, G. 2002, A\&A, 381, L37

Tully, R. B., \& Fisher, J. R. 1977, A\&A, 54, 661

Turner, A., Ferrarese, L., Saha, A., et al. 1998, ApJ, 505, 207 (Tur98)

Udalski, A., Szymanski, M., Kubiak, M., et al. 1999, Acta Astron. 49, 201 (Uda99)

Walker, A. R. 1988, PASP, 100, 949 (Wal88) 